\title{
Anisotropia sísmica do manto superior com a divisão de ondas cisalhantes SKS e SKKS nas regiões Norte e Centro-Oeste do Brasil
}

Lyara Villanova Silverio; Marco Antônio Gonzaga de Siqueira; George Sand França; Cristobal Condori Quispe. Observatório Sismológico - Instituto de Geociências, UnB

Copyright 2018, SBGf - Sociedade Brasileira de Geofísica

Este texto foi preparado para a apresentação no VIII Simpósio Brasileiro de Geofísica, Salinópolis, 18 a 20 de setembro de 2018. Seu conteúdo foi revisado pelo Comitê Técnico do VIII SimBGf, mas não necessariamente representa a opinião da SBGf ou de seus associados. É proibida a reprodução total ou parcial deste material para propósitos comerciais sem prévia autorização da SBGf.

\section{Resumo}

O método de divisão de ondas cisalhantes vem sendo aplicado para estudar a anisotropia no manto superior em algumas estações sismográficas nas regiões Norte e Centro-Oeste do Brasil, onde o usamos com o objetivo de entender a direção do fluxo astenosférico. A dependência direcional da propagação da onda sísmica está fortemente relacionada com o alinhamento preferencial (LPO) da olivina, parâmetro indicador de processos geodinâmicos das deformações do manto associado aos regimes passados e atuais. A área de estudo é composta de uma estrutura tectônica que é dominada pelo Cráton Amazônico, uma das áreas mais expressivas do período Arqueano-Proterozóico, que ainda apresenta pequeno conhecimento geofísico e geológico. Para a análise, foram utilizadas as fases SKS e SKKS de eventos ocorridos entre 2014 e $2017 \quad\left(88^{\circ}<\Delta<130^{\circ}\right)$, com magnitude igual/superior a 5,5 Mw, todos detectados em estações da Rede Sismográfica Brasileira (RSBR - UnB). Para medições dos parâmetros de anisotropia sísmica, determina-se a direção de polarização rápida $(\Phi)$ e o tempo de atraso $(\delta \mathrm{t})$ entre a direção de polarização rápida e a lenta, utilizando três técnicas: mínima-energia, rotação-correlação e o autovalor. Neste trabalho encontramos resultados onde as direções de polarização rápida estão orientadas quase perpendicularmente ao modelo de fluxo teórico de Conrad na região do Cráton Amazônico e aproximadamente paralelas nas outras estações, sendo, então, proposto que a anisotropia nessas regiões esteja relacionada a concordâncias entre as evidências geológicas e sísmicas para a localização da borda do Cráton.

\section{Introdução}

A anisotropia sísmica manifesta-se como a variação de uma ou mais propriedades físicas de acordo com a direção de propagação das ondas sísmicas, devido às orientações preferenciais que adquirem os minerais constituintes das rochas na trajetória de propagação. As orientações preferenciais da estrutura cristalina interna e externa refletem o esforço ao qual foram submetidos os cristais. Meios anisotrópicos foram observados e estudados na crosta superior da Terra, causada por micro fraturas (Crampin \& Chastin, 2003); no manto superior, devido à direção de alinhamento preferencial do mineral olivina (Tommasi, 1998); na zona de transição do manto
(Tommasi et al., 2004); no manto inferior (Wookey et al., 2002) e na camada D" (Savage, 1999).

O estudo da anisotropia sísmica é importante para compreender a relação entre a deformação e o fluxo do manto superior (Andrade, 2008) e pode proporcionar informações sobre o interior do planeta, uma vez que as ondas sísmicas são sensíveis às mudanças físicas e cristaloquímicas dos materiais em sua trajetória de propagação. Uma das técnicas mais implantadas para estudo de anisotropia utiliza as ondas cisalhantes. Especificamente, este trabalho tem o objetivo de estudar a relação entre a direção de maior velocidade da onda $S$ (Vs) com as direções do fluxo do manto litosférico no Norte e Centro-Oeste do Brasil, calculado a partir do estudo de ondas SKS e SKKS. Essas fases foram escolhidas sabendo-se que as mesmas tem polarização radial depois da conversão de onda $P$ para onda $S$ no limite Manto-Núcleo, onde as vibrações na componente transversal são interpretadas como efeito da divisão da onda cisalhante. Uma vez que a onda passa pelo núcleo, sua incidência é aproximadamente vertical na superfície, possibilitando considerar que toda a anisotropia medida está logo abaixo da estação sísmica (Silver \& Chan, 1991). Os primeiros $410 \mathrm{~km}$ do manto superior são compostos primordialmente dos minerais olivina, enstatita e diopisídio (Buontempo, 2008), sendo que a anisotropia sísmica está fortemente ligada à orientação cristalina preferencial (LPO, do inglês lattice preferred orientation) da olivina, o mais anisotrópico dos três e o mais abundante no manto, formando aproximadamente $90 \%$ de sua composição (Andrade, 2008).

A LPO pode ser devido ao deslizamento da Litosfera sobre a Astenosfera, estar relacionada à direção de maior esforço de antigos processos orogênicos ou a combinação dos dois processos (Silver et al., 1994) e ainda está diretamente ligada ao tipo de olivina, que varia de acordo com as condições do meio. Para os efeitos de discussão dos resultados, espera-se que os eixos sísmicos rápidos estejam orientados paralelamente a direção do fluxo do manto (Jung et al., 2006; Long, 2009b), alinhados a direção do movimento relativo de placa entre as placas primordiais e em subducção. Neste caso, o fluxo do manto é bidimensional (2D). Por causa de seu ângulo de incidência aproximadamente vertical, as medidas de anisotropia SKS são apenas sensíveis ao componente horizontal do fluxo do manto superior. O movimento de placa induz um fluxo turbulento em torno das bordas laterais, dentro de um plano aproximadamente horizontal (Schellart, 2004; Stegman et al., 2006). O rollback da placa conduz um fluxo de retorno 3-D no manto logo abaixo da placa e uma barreira, provavelmente no topo ou no fundo da zona de transição, mantém o manto fluindo sob a placa (Russo e Silver, 1994; Savage, 1999; Schellart, 2004; Long and Silver, 2008, 2009b). Tal barreira pode ser devida ao aumento 
da viscosidade no limite superior do manto inferior ou à alta pressão abaixo da placa produzida pela placa em subducção (Schellart, 2004).

A anisotropia sísmica do manto superior é estudada com dados adquiridos em superfície mediante a divisão das ondas S, que, quando passam por um meio anisotrópico em subsuperfície, separam-se em dois componentes polarizados ortogonais, dependentes de sua direção de propagação (Figura 1), fenômeno definido como divisão da onda cisalhante (Savage, 1999). A direção de polarização com maior velocidade de propagação chamase direção de polarização rápida e a que tem menor velocidade, polarização lenta. A LPO dos cristais anisotrópicos é a direção onde a onda que foi dividida tem a velocidade de propagação maior. Então, o estudo da divisão das ondas $S$ consiste na busca dos dois parâmetros causadores da anisotropia: 1) O atraso temporal $(\delta \mathrm{t})$ entre as fases rápida e lenta, relacionado com a espessura e a anisotropia intrínseca do meio anisotrópico e 2) A orientação $(\varphi)$ dos planos de polarização, relacionada com a orientação da estrutura do meio.

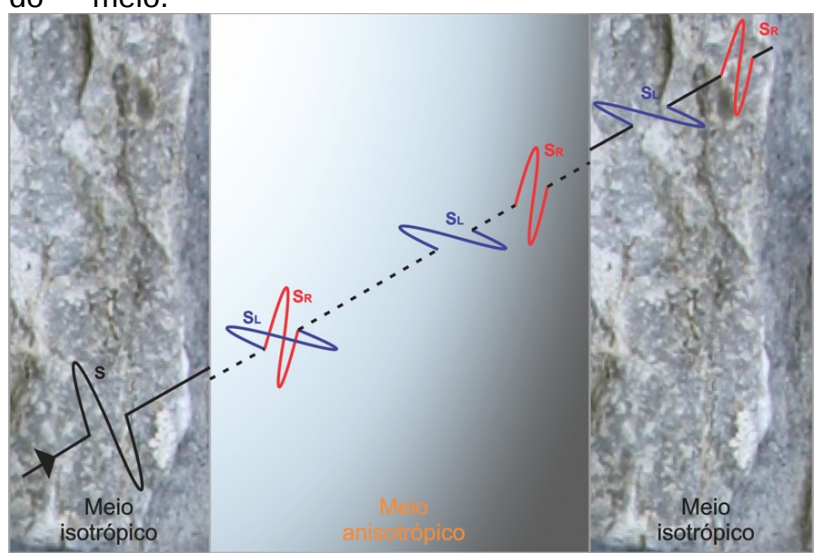

Figura 1: A divisão da onda ocorre ao passar por um meio anisotrópico, apresentando dois parâmetros: O atraso temporal $(\delta \mathrm{t})$ entre as fases rápida $(\mathrm{SR})$ e lenta $(\mathrm{SL})$ e a orientação $(\varphi)$ dos planos de polarização, perpendiculares entre si (representados em vermelho e azul).

Quando a anisotropia está localizada na litosfera, os parâmetros de divisão ( $\delta$ t e $\varphi$ ) podem estar relacionados com uma deformação antiga ou ativa, enquanto que se a mesma ocorre na Astenosfera, $\delta$ t e $\varphi$ podem ser atribuídos ao fluxo mantélico atual, produzido pelo movimento diferencial entre as placas litosféricas e o manto subjacente.

\section{Metodologia}

Foram analisados sismogramas com o programa Splitlab (Wüstefeld et al., 2007), que é executado no MatLab $b_{T M}$, de 15 estações da Rede Sismográfica Brasileira (RSBR), entre o período de janeiro de 2014 a novembro de 2017 (Figura 2).

O processamento com o SplitLab aplica 3 técnicas nos dados para remover o efeito de divisão (assim encontrando $\varphi$ e $0 \quad \delta \mathrm{t}$ ): 0 primeiro é o método de rotação-correlação (RC), que usa a maximização do coeficiente de correlação cruzada entre as componentes radial $(R)$ e transversal $(T)$ das formas de onda na janela selecionada; o segundo é o método de mínima energia, que consiste em determinar a direção de polarização rápida e o intervalo de tempo entre as divisões da onda, rotacionando para componentes $R$ e $T$ e minimiza a energia na componente $T$, após a correção da anisotropia; e o terceiro é o método de autovalor, que usa o azimute reverso (backazimute) como polarização inicial ou para estimar o movimento das partículas após a correção da anisotropia e linearização da forma de onda. Todos os três métodos realizam uma pesquisa em grade em função dos parâmetros $\varphi$ e $\delta$ t, que melhor removam o efeito de divisão de onda, linearizando o movimento da partícula em plano NE ou QT (Wustefeld et al., 2008).

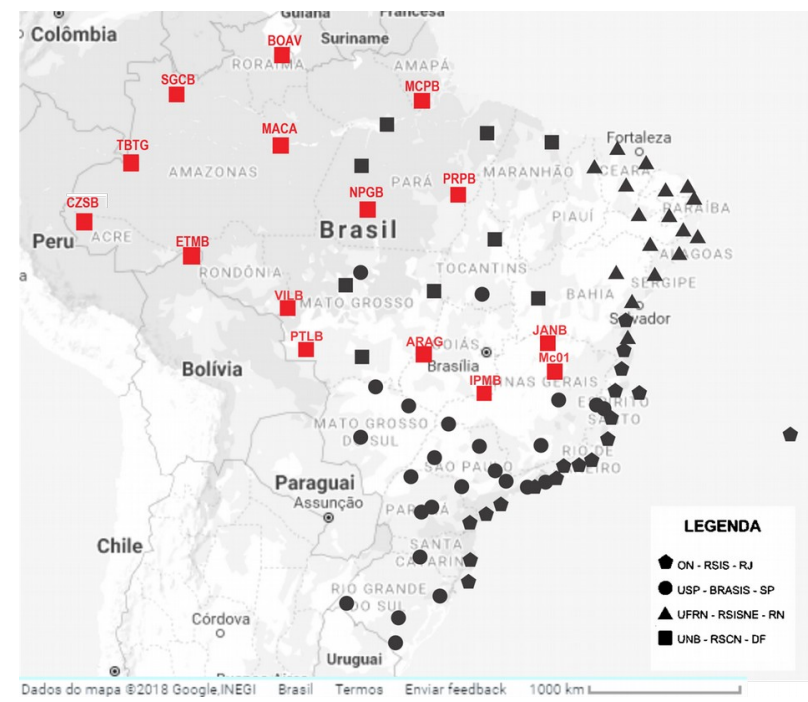

Figura 2: Estações sismográficas da RSBR - Rede Sismográfica Brasileira, em vermelho as estações da rede BR (UnB - RSCN) usadas neste estudo.

Os eventos usados foram com distâncias epicentrais entre $88^{\circ}$ e $130^{\circ}$ e com magnitude maior ou igual a 5,5 Mw (Figura 3), para que as fases SKS e SKKS tivessem energia suficiente para serem distinguidas do ruído nos sismogramas processados. Para minimizar o efeito de ruído, foi usado filtro com a banda $0,03 \mathrm{~Hz}-0,3 \mathrm{~Hz}$.

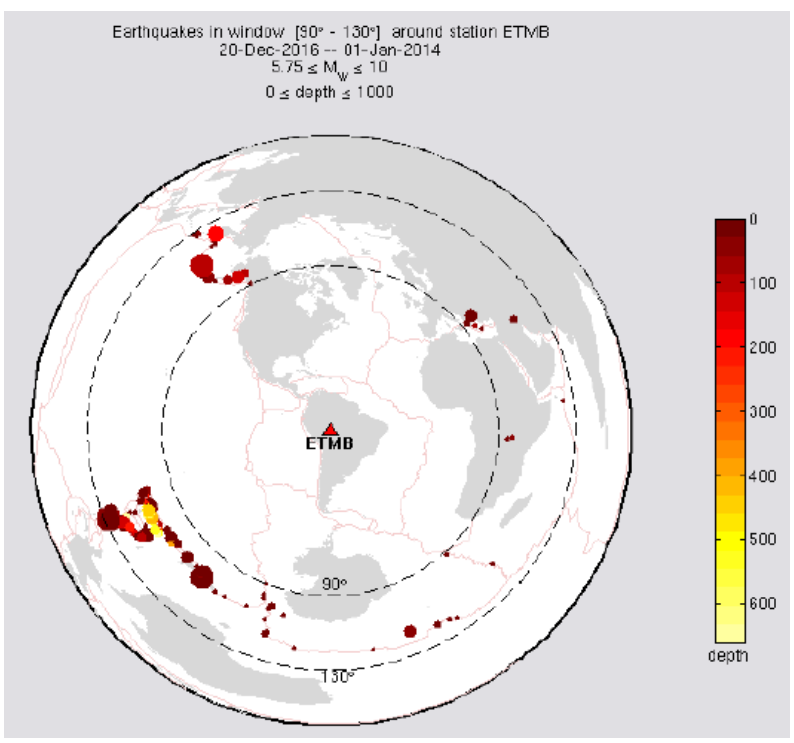


Figura 3: Distribuição espacial dos terremotos usados neste estudo. A projeção está centralizada no Brasil (estação ETMB). Ao redor, os dois círculos delimitam a distância epicêntrica entre $88^{\circ} \mathrm{e} 130^{\circ}$. Imagem gerada no SplitLab, em 16/12/2017 as 13:47.

\section{Resultados}

Os resultados de cada evento foram classificados de acordo com a qualidade dos dados, sendo "good" para dados bons, onde a componente transversal é bem visível e a correção da energia mínima (método SC) fornece boa correção do movimento da partícula de movimento elíptico para movimento linear; "fair", quando o dado possui informações relevantes, porém com incerteza em um ou mais fatores de correção; e "poor", quando o dado apresenta alta incerteza, que neste trabalho não foram utilizados. De forma geral os resultados estão na tabela 1 e figura 4.

Tabela 1: Resultados preliminares

\begin{tabular}{|c|c|c|}
\hline Estações & $\varphi$ & $\delta \mathrm{t}$ \\
\hline ARAG & 71 & 1,75 \\
\hline BOAV & $-65,5$ & 0,45 \\
\hline ETMP & 71,33 & 0,87 \\
\hline IPMB & -78 & 0,83 \\
\hline JANB & 70.25 & 0,8 \\
\hline MACA & $-64,36$ & 0,81 \\
\hline MC01 & 67,5 & 1,5 \\
\hline MCPB & 76,71 & 1,0 \\
\hline PDRB & $-64,95$ & 1,08 \\
\hline PRPB & 75,83 & 1,12 \\
\hline SGCB & 88 & 0,9 \\
\hline VILB & $-31,23$ & 1.03 \\
\hline TBTG & $-71,4$ & 1,16 \\
\hline CZSB & 21,3 & 0,73 \\
\hline NPGB & 73,86 & 1 \\
\hline & & \\
\hline & & \\
\hline
\end{tabular}

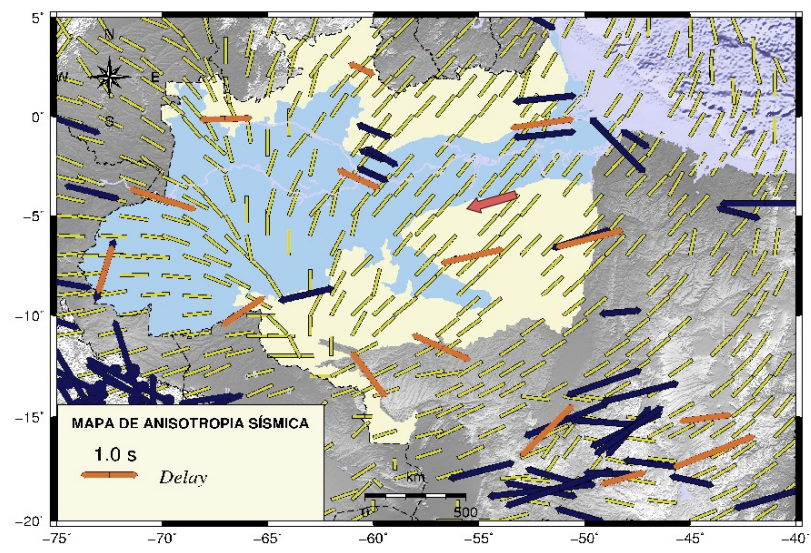

Figura 4: Resultados prévios dos parâmetros da anisotropia sísmica. Em azul, as medições compiladas, em laranja a média compilada para cada estação processada e em amarelo os valores de direção do fluxo mantélico teórico proposto por Conrad (2007).

\section{Discussão e Conclusões}

Os resultados compilados mostram direções de polarização rápida orientadas quase perpendicularmente ao modelo de fluxo teórico de Conrad (2007) na região do Cráton Amazônico e aproximadamente paralelas nas outras estações (Figura 4). É possível que a anisotropia nessas regiões esteja relacionada com concordâncias entre as evidências geológicas e sísmicas para a localização da borda do Cráton, no presente estudo, que a orientação do eixo de polarização é controlada pelo fluxo do manto astenosférico ao redor da borda do Cráton.

As estações processadas e estudadas oferecem boa cobertura da geometria da área e as medidas de divisão demonstraram relação entre o fluxo do manto, a geologia histórica e a geodinâmica nas regiões Norte e CentroOeste do Brasil. As condições físicas abaixo da região de estudo possivelmente apresentam baixo estresse, baixo teor de água e temperatura relativamente alta, prevendose olivina de tipo A (Jung et al., 2006; Long e Silver, 2008). Os eixos rápidos mudam de orientação na borda do Craton e alinham perpendicularmente ao movimento teórico de placa de Conrad, comparados na Figura 4, apresentando características de olivina de tipo $\mathrm{B}$. $\mathrm{Na}$ zona de subducção entre as Placas de Nazca e Sul Americana, altas temperaturas e desidratação da placa ocorrem em profundidades de até $150 \mathrm{~km}$ (Manea e Manea, 2011), inferindo presença de olivina do tipo $C$ nas bordas. Relembrando que olivinas de tipo $A$ e $C$ possuem eixos rápidos que se alinham na direção do fluxo do manto, podendo-se inferir que as mudanças nos os eixos de polarização na região do Cráton Amazônico possuem forte influencia resultante de parâmetros dinâmicos que interferem diretamente na física do material em subsuperfície presente na trajetória da onda sísmica medida, sendo: A presença de fluxo 2-D e 3-D no manto; mudanças na morfologia da zona de subducção e intercontinental (Apesar de que a mudança nas direções rápidas de polarização SKS estar razoavelmente próxima a direções perpendiculares, são indicativas do fluxo sublitosférico); dependência azimutal atrasada, indicação de que duas ou mais camadas anisotrópicas estão presentes sob a estação. Levando, ainda, em consideração o padrão de anisotropia da onda superficial nos 200 quilômetros superiores da Terra, a fraca resolução de profundidade proporcionada pelas medidas de camadas de divisão da onda de cisalhamento e que a anisotropia SKS estaria localizada aproximadamente a uma profundidade de 200 a $400 \mathrm{~km}$, provavelmente correspondente a Astenosfera inferior, a direção anisotrópica pode ser afetada pelo movimento da placa (Stubailo, 2015), explicando por que a direção rápida está alinhada com o movimento da placa de Sul Americana na região estudada, excetuando a região do Cráton Amazônico, que possui diferenças morfológicas e de espessura. Por fim, o presente estudo de anisotropia sísmica no Brasil contribui com informações que podem ser somados aos trabalhos de anisotropia já realizados em todo o país e na América do Sul, auxiliando a um 
melhor entendimento da geodinâmica e de características geofísicas regionais e/ou continentais.

\section{Agradecimentos}

Agradecemos a CAPES, a Rede Sismográfica Brasileira, RCBR, CNPq - através do Projeto Estudos tectônicos sobre a bacia do Parecis e ao Observatório Sismológico da Universidade de Brasília pelo apoio.

\section{Referências}

Andrade, M. G. (2008). Anisotropia sísmica do manto superior na América do Sul com a divisão de ondas SKS. Dissertação de mestrado - USP - São Paulo/SP.

Buontempo, L. (2008). Anisotropía sísmica en la Península Ibérica. Tesis doctoral - Universidad de Granada - Granada - Spain.

Conrad, C. P.; Behn, M. D.; Silver, P. G. Global mantle flow and the development of seismic anisotropy: Differences between the oceanic and continental upper mantle. Journal of Geophysical Research (Solid Earth), v. 112, p. $7317+$, jul. 2007.

Crampin, S., Chastin, S. (2003). A review of shear wave splitting in the crack-critical crust. Geophysical Journal International, v.155:221-240.

Karato S.-i., Jung H., Katayama I., Skemer P., 2008, Geodynamic significance of seismic anisotropy of the upper mantle: New insights from laboratory studies, Annu. Rev. Earth Planet. Sci., 36, 59-95, doi:10.1146/ annurev.earth.36.031207.124120.

Kneller E.A, van Keken P.E., Karato S.-i., Park J., 2005, B-type olivine fabric in the mantle wedge: Insights from high-resolution non-Newtonian subduction zone models, Earth Planet. Sci. Lett., 237, 781-797, doi:10.1016/j.epsl.2005.06.049.

Jung H., Karato S.-i., 2001, Water-induced fabric transitions in olivine, Science, 293, 1460-1463.

Jung H., Katayama I., Jiang Z., Hiraga T., Karato S., 2006, Effect of water and stress on the lattice-preferred orientation of olivine, Tectonophysics, 421, 1-22.

Long M.D., Silver P.G., 2008, The subduction zone flow field from seismic anisotropy: A global view, Science, 319, 315-318, doi:10.1126/science.1150809.

Long M.D., Silver P.G., 2009a, Shear wave splitting and mantle anisotropy: Measurements, interpretations, and new directions, Surv. Geophys., 30, 407-461, doi:10.1007/ s10712-009-9075-1.

Long M.D., Silver P.G., 2009b, Mantle flow in subduction systems: The subslab flow field and implications for mantle dynamics, J. Geophys. Res., 114, B10312, doi:10.1029/2008JB006200.

Long M.D., 2013, Constraints on subduction geodynamics from seismic anisotropy, Rev. Geophys., 51, 76-112, doi:10.1002/ rog.20008.

Manea V.C., Manea M., 2011, Flat-slab thermal structure and evolution beneath central Mexico, Pure Appl. Geophys., 168, 1475- 1487, doi:10.1007/s00024-0100207-9.
Paczkowski K., Thissen C.J., Long M.D., Montési L.G.J., 2014b, Deflection of mantle flow beneath subducting slabs and the origin of subslab anisotropy, Geophys. Res. Lett., 41, 6734-6742, doi:10.1002/2014GL060914.

Russo R.M., Silver P.G., 1994, Trench-parallel flow beneath the Nazca plate from seismic anisotropy, Science, 263, 1105-1111.

Savage, M. K. (1999). Seismic anisotropy and mantle deformation. What have we learned from shear wave splitting? Reviews of Geophysics, v.37:65-106.

Schellart W.P., 2004, Kinematics of subduction and subduction-induced flow in the upper mantle, J. Geophys. Res., 109, B07401, doi:10.1029/2004JB002970.

Stegman D.R., Freeman J., Schellart W.P., Moresi L., May D., 2006, Influence of trench width on subduction hinge retreat rates in 3-D models of slab rollback, Geochem. Geophys. Geosyst., 7, Q03012, doi:10.1029/2005GC001056.

Silver, P. G. and W. W. Chan (1991). Shear wave splitting and subcontinental mantle deformation. Journal of Geophysical Research, v.96.

Silver, P. G., Savage and M. K. (1994). The interpretation of shear-wave splitting parameters in the presence of two anisotropic layers. Geophysical Journal International, v.119:949-963.

Stubailo I., 2015, Seismic anisotropy below Mexico and its implications for mantle dynamics, Ph. D. thesis, 119 pp., University of California, Los Angeles, CA, USA.

Tommasi, A. (1998). Forward modeling of the development of seismic anisotropy in the upper mantle. Earth and Planetary Science Letters, v.160.

Tommasi, A., Mainprice, D., Cordier, P., Thoraval, C., Couvy, H. (2004). Strain-induced seismic anisotropy of wadsleyite polycrystals and flow patterns in the mantle transition zone. Journal of Geophysical Research, v.109.

Wookey, J., J. M. Kendall and G. Barruol (2002). Midmantle deformation inferred from seismic anisotropy. Nature, v.415:777-780.

Wüstefeld, A., Bokelmann, G. H. R. (2007). Null detection in shear-wave splitting measurements. Bull. Seismol. Soc. Am., v.97:1204-1211.

Wüstefeld, A., Bokelmann, G. H. R., Zaroli, C., Barruol, G. (2008). SplitLab: A shear-wave splitting environment in Matlab. Computers \& Geosciences, v.34:515-528. 